\title{
DIESEL FUEL ADDITIVES: WORTH CONSIDERING? LOCAL EXHAUST EMISSIONS AND FUEL CONSUMPTION TEST RESULTS
}

\author{
Palo Trevisan ${ }^{182}$, Nico Claassen ${ }^{2}$ \\ ${ }^{1}$ Clean Air Testing Solutions cc, pawutrevi@gmail.com \\ ${ }^{2}$ School of Health Systems and Public Health, Faculty of Health Sciences, University of Pretoria, \\ nico.claassen@up.ac.za
}

\begin{abstract}
Pollutant regulations are not a matter of concern only for engine manufacturers but they also require policy implementation, commitment and a dedicated effort by industry. In particular, some in the mining industries are expanding their efforts to provide environmentally friendly working procedures and conditions. Use of fuel enhancers has been suggested. The Letšeng Diamond Mine, in Lesotho has taken the lead and commissioned a study on three different fuel additives. For all three fuel additives, three prominent variables were measured, namely diesel fuel consumption, diesel exhaust gases and smoke emission levels. All instruments used were EPA certified and calibrated or verified according to the specification of the manufacturer and or international best practice to ensure accurate readings. This paper discusses the results obtained on site over a thirteen month study period involving different classes of machinery and under different weather conditions. Product $C$ improved fuel consumption by $9.9 \%$ and reduced black smoke by $32 \%$. Pitfalls have been identified with product $A$ and product $B$ that can help to avoid costly trials on other sites, such as rusted and blown injectors, engine failure and corroded seals, valves and cylinders. Further research is required however to determine if these additives will become major role players to reduce emissions and improve efficiency. In conclusion, we cannot ignore the use of fuel additives when talking about diesel pollution and the fact that the additives cou Id assist in reducing harmful emissions even if only one additive appears to be performing positively in terms of consumption and emissions, such as product $\mathrm{C}$. Three strategies have been identified for reducing emissions on diesel earth moving equipment currently in use: engine modifications, exhaust gas treatment and fuel composition modifications. Engine modifications and exhaust gas treatment are considered costly alternatives to achieve reduced exhaust emissions plus these strategies are proven to have little or no positive impact on fuel economy performance. The study has shown one factor clearly - we cannot ignore the effects of additives both with regard to emissions and combustion efficiency.
\end{abstract}

Keywords: Diesel, fuel, combustion, Emissions.

\section{Introduction}

One of the major polluting contributors to our environment today is the internal combustion engine, either in the form of spark ignition petrol or diesel versions. In parallel to this serious environmental threat, the main source of fuel for these engines, namely crude oil, is being depleted at high rates, so that the development of less polluting and more efficient engines is today of extreme importance for engine manufacturers [Rakopoulos et al., 2004]. Pollutant regulations are a matter of concern for engine manufacturers but they require also an important effort by the fuel industry to provide environmentally friendly fuels. These fuels will help to achieve reduced emissions and improve the performance of new engine and after-treatment technologies [Wong et al., 1985].

The interest in researching alternatives to diesel fuel has been growing due to the increasing concern over the cost and supply of petroleum fuels. From this interest, there are two imperative issues that need to be solved. One is to find alternative fuel sources that can reduce the dependence on petroleum fuels. The other equally important issue is to evaluate the performance of these alternative fuels and whether they can reduce particulate matter (PM) and gaseous emissions [Armas et al., 2010].

There is an increasing urgency in finding ways to improve fuel economy of motor vehicles while controlling tailpipe emissions to meet ever-tighter standards. Diesel engines have the advantages of better fuel economy, lower emissions of hydrocarbons ( $\mathrm{HC}$ ) and carbon monoxide (CO). However, diesel engines cause high emissions of PM and nitric oxides (NOx), and it is hard to reduce both simultaneously [Yanfeng et al., 2006].

Three strategies for reducing emissions from engines can be followed: engine modifications, fuel composition modifications or exhaust gas treatment. The distinction between PM and $\mathrm{HC}$ emissions is to a large extent a matter of condensation temperature. 
The conversion of fuel to unburnt particulates is most likely to occur when the engine is operated at a high load, where the volume of fuel injected is high and the time available for combustion is short [Sarvi et al. , 2008].

Methods to reduce PM and NOx emissions include high-pressure injection, turbo charging and exhaust after treatments. Fuel additives are still investigated and thought to be one of the attractive solutions. Adding oxygenates in diesel can substantially reduce emissions of PM without significant effects on NOx. Oxygenates are also full or partial substitutes for diesel [Yanfeng et al., 2006)]. In this paper, Product A, $B$ and $C$ are being compared as diesel additives according to the following criteria: ease of blending with diesel; availability of abundant sources for synthesis; and little change in the fuel delivery system, engine power or fuel consumption.

Today in Europe, only smoke exhaust measurements are used to characterise diesel engine pollution. Because of measurement equipment, estimation of soot particles presented in the exhaust gas depends on optical properties. There is not as yet an on-line sensor used to characterise diesel engine pollution. The objective is twofold. Firstly, exhaust emissions outputs, in terms of harmful emissions. Secondly, experimental measurements are made to determine diesel particle density before and after addition of products $A, B$ and $C$ respectively and the respective associated fuel consumption improvement figures [Ouenou-Gamo et al., 1998].

Previous studies investigated the influence of basic engine components and design parameters on the emissions from large diesel engines [Sarvi \& Zevenhoven, 2009]. For modern engines these parameters include fuel injection timing (SOI), compression ratio (CR), fuel injector design, and more. These parameters are important for the control of fuel economy and exhaust emissions (NOx, CO, $\mathrm{HC}$, soot (smoke) and particulate matter (PM)), while maximizing power output, efficiency and performance [Sarvi \& Zevenhoven, 2009].

In essence, diesel fuel additives are used to improve the efficiency and performance of diesel fuel. This is achieved by increasing the temperature and speed at which the fuel burns so that more energy is produced by the diesel fuel. Because of this, a significant improvement in fuel economy is also observed. Improvements in acceleration, power and torque are noticed in varying degrees, depending on the additive product used. Because the engine uses the fuel more efficiently, a reduction in exhaust emissions such as carbon monoxide and other gases that are harmful to the environment will be noticed. Besides causing fewer problems to the environment, this also helps the engines pass the strict Environmental Protection Agency (EPA) emission requirements. Diesel fuel additives also improve the lubricity of the fuel, which reduces wear and tear in the engine and other components such as fuel pumps and fuel injectors. In the long run, the engine will serve longer saving even more money. For cold areas, or prolonged winter seasons, diesel fuel additives helps prevent diesel from clouding and producing waxy substances that can clog up the engine.

After taking into account all the benefits of diesel fuel additives to the engine and the environment, particularly the savings in fuel costs and repair costs, as well as the performance gains experienced, the use of these additives could prove to be cost effective and an excellent investment. The next question is which fuel additive provides the best performance for specific diesel engines.

The aim of the present work was therefore to perform a comparative study to determine the effect of fuel enhancing additives, mixed with $500 \mathrm{ppm}$ sulphur diesel, on the emissions and on consumption. Experimental work aimed at fuel economy and low pollutant emissions from diesel engines includes successive changes of each of the many parameters involved, which is very demanding in terms of money and time. Today, the development of powerful digital computers leads to the alternative of simulation of engine performance by a mathematical model. In these models, the effects of various designs and operation changes can be estimated in a fast and non-expensive way, provided that the main mechanisms are recognized and correctly modelled [Rakopoulos et al., 2004].

\section{Methodology}

Product A was a locally blended alcohol based product. Product $B$ was a locally blended paraffin based product. Product $C$ was a USA blended marine seal oil based product, with EPA certification. Product $C$ was certified by major engine manufacturers, namely CAT, Cummins, Volvo, Mercedes, Landrover.

Testing on the three diesel fuel additives was conducted between March 2009 and March 2010, according to the standardised EPA protocol for cumulative effect fuel additive testing for diesel fuels, using an EPA compliant Autologic Gas analyser, and the standard methodology for snap acceleration testing, performed with the Autologic Opacity meter, SAE J1667, according to manufacturer's specifications. Consumption testing was performed by making use of the calibrated electronic fuel management system (Petroman Pacs running on a SQL database).

The fuel consumption baseline for all the earth moving equipment was based on the electronic data collected from the onsite fuel management system. This data was supported with manual documentation for two weeks prior to starting the project. These data were used as the standard for comparison for collected data after addition of the diesel fuel additives were initiated. Exhaust emissions were monitored onsite before initiation of the diesel fuel additives. This was repeated three times prior to addition of the products. These values were 
standardised as the baseline and were used as a comparison for data collected after initiation of the product.

The Autologic gas analyser was used to monitor the emissions on all the earth moving equipment. The Autologic gas analyser and the Autologic heavy-duty opacity meter were used on the fixed-head dump trucks at Letšeng Diamond Mine, present at the time of running the project. All earth moving equipment was dosed onsite, during morning and evening refuelling. Dosing with all the diesel fuel additives were on a ratio specified by the suppliers. Product A \& $B$ had a dosing ratio of $4 \mathrm{ml}$ of product per 1 litre of diesel, while Product $C$ had a dosing ratio of $3.2 \mathrm{ml}$ of product per 1 litre of diesel. The earth moving equipment was dosed before refuelling based on the amount of time they worked. By making use of the current average consumption in litres per hour of the individual vehicles, product volumes were estimated and added to the vehicles. The vehicles were refuelled and any difference in calculated litres and actual litres put in was accounted for by topping up with more product. Product was added to the fuel tank before refuelling, to enhance mixing. All fuel for the excavators and dozers was dispensed out of an accurately calibrated diesel bowser unit number 2 , while the fixed-head dump trucks were refuelled at pump 3 of the calibrated diesel fuel depot. The electronic meter on the bowser and pump 3 was used to obtain diesel volumes put into the vehicle fleets. Fuel filters were changed after 7 days of product initiation, as guided by the standardised EPA fuel testing protocol.

When using an emissions analyser, exhaust gas readings are obtained by inserting a small probe into the tailpipe while the engine is running. Exhaust emissions were pulled into the analyser by a small fan. Most units use an infrared light to check for $\mathrm{HC}$, $\mathrm{CO}$ and $\mathrm{CO}_{2}$ in the exhaust. Depending on the degree of light absorbance and diffusion, the analyser calculates the percentage of each gas present in the sample and displays a value for each gas. An electrochemical sensor is used to measure the amount of $\mathrm{O}_{2}$ and $\mathrm{NO}_{x}$ in the exhaust air (tail pipe). To assure accurate readings, analysers were verified using a standard calibration gas at the factory. This may have to be done periodically depending on the requirements of the unit. A calibration gas was also used before and after each test was performed with the Autologic gas analyser because the high altitude of the mine was a concern. A leak check test was performed on the emissions analyser at the beginning and end of each test period.

When using an opacity meter, the nozzle of the meter is mounted on a stack or duct and projects a beam of light from an optical head on one side to a reflector on the other. The light is reflected back through the gas (double pass for greater sensitivity) to the optical head. The detector in the optical head determines opacity by comparing the ratio of the energy level of the reflected light to the energy level of the projected light.

A Super Wide Band Diode (SWBD) generates the transmitted light. Due to the SWBD's broadband nature, it is not influenced by other light sources (like sunlight), and is measurement results are not influenced by temperature or other factors.

\section{Results}

Table 1 details the consumption results of product $A$. The measurements were performed over a period of a week. The reduction in amount of fuel used, known as "consumption improvement" was 2 - 3\% over the range of the Caterpillar articulated dump trucks.

Fuel consumption and exhaust emission results are not available for product $B$ as the product failed on mechanical issues. The tests started in the same manner as for product $\mathrm{A}$ and $\mathrm{C}$, according to EPA protocol. On pouring the product into the fuel system, it was very thick and glue-like. This product did not mix with the fuel and within an hour of adding the product $B$ to the system, there was severe injector failure. Due to this premature failure, the product could not be tested and brought about very severe doubts about this type of product being tested. The mine subsequently discontinued use of the product without further discussions.

Tables 2, 3 and 4 are the results of product $C$. The results were very encouraging early on in the study. The mine decided to proceed with further detailed testing in order to see the true long-term effects of this product on various vehicle fleets as the short- term results corresponded with the manufacturer's claim. As per manufacturer's statement, the first ten days of the product being introduced into the fuel system was classified as the "clean-up" phase. Tabel 1 on next page.

This was based on the detergent and algaecide properties of the product cleaning out the fuel tank and fuel lines, dissolving and pushing all contaminants into the fuel filter. The fuel filter was changed after ten days on each of the vehicles, to allow uninhibited fuel flow. Once the filters were changed, the results phase was established. The results of the last ten days of the study were most significant and reflective of the manufacturer's claims. Tables 2, 3 and 4 display the remarkable improvements in consumption and exhaust emissions. Tables on the next page. 
Table 1: Summary table from phase 1 of the fuel enhancement evaluation at Letšeng Diamond Mine, showing the fuel economy figures in litres per hour of the caterpillar articulated dump trucks before and after initiation of Product $A$.

\begin{tabular}{|c|c|c|c|c|c|}
\cline { 2 - 4 } \multicolumn{1}{c|}{} & Baseline & \multicolumn{3}{c|}{ Results } & \multirow{2}{*}{$\begin{array}{c}\% \\
\text { change to } \\
\text { baseline }\end{array}$} \\
\cline { 1 - 4 } Unit & LPH & Hours & Litres & LPH & \\
\cline { 1 - 4 } 1010010 & $\mathbf{2 5 . 2 8}$ & 120.3 & 2980.8 & $\mathbf{2 4 . 7 7}$ & $\mathbf{- 2}$ \\
\hline 1010011 & $\mathbf{2 8 . 3 1}$ & 73.4 & 2265.9 & $\mathbf{3 0 . 8 9}$ & $\mathbf{+ 8}$ \\
\cline { 1 - 4 } 1010012 & $\mathbf{2 8 . 2 0}$ & 79.3 & 2463.3 & $\mathbf{3 1 . 0 7}$ & $\mathbf{+ 9}$ \\
\cline { 1 - 4 } AVERAGE & $\mathbf{2 7 . 2 6}$ & 273.0 & 7710.0 & $\mathbf{2 8 . 9 1}$ & $\mathbf{+ 6}$ \\
\hline
\end{tabular}

Table 2. Summary table from phase 1 of the fuel enhancement evaluation at Letšeng Diamond Mine, showing the fuel economy figures in litres per hour of the caterpillar excavators before and after initiation of Product $\mathrm{C}$.

\begin{tabular}{|c|c|c|c|c|c|c|c|c|c|}
\hline \multirow[b]{2}{*}{ Unit } & \multirow{2}{*}{$\begin{array}{c}\text { Baseline } \\
\text { LPH } \\
\end{array}$} & \multicolumn{3}{|c|}{ First 10 Days } & \multicolumn{3}{|c|}{ Last 10 Days } & \multirow{2}{*}{$\begin{array}{c}\text { Change } \\
\text { to } \\
\text { baseline }\end{array}$} & \multirow{2}{*}{$\begin{array}{c}\% \\
\text { Change to } \\
\text { first } 10 \\
\text { days }\end{array}$} \\
\hline & & Hours & Litres & LPH & Hours & Litres & LPH & & \\
\hline $365 / 01$ & 37.7 & 239 & 9258 & 38.7 & 219 & 7614 & 34.8 & $-7.7 \%$ & $-10.2 \%$ \\
\hline $365 / 02$ & 39.7 & 170 & 6713 & 39.4 & 226 & 8477 & 37.5 & $-5.5 \%$ & $-4.8 \%$ \\
\hline $385 / 01$ & 58.0 & 232 & 13309 & 57.3 & 192 & 9626 & 50.3 & $-13.3 \%$ & $-12.2 \%$ \\
\hline $385 / 03$ & 54.2 & 220 & 12921 & 58.9 & 220 & 12724 & 57.8 & $6.6 \%$ & $-1.8 \%$ \\
\hline AVERAG E & 48.6 & & & 49.0 & & & 44.9 & $-5.0 \%$ & $-8.4 \%$ \\
\hline
\end{tabular}

Note 1: Since the baseline fuel consumption information of the electronic system and the manual recording system had a large variance, the results of the final stage of phase 1 were compared to the first 10 days of testing, as opposed to the baseline, as this recording of consumption data was reliable and valid. The comparison to the baseline is represented for interest but is not used in the report.

Note 2: $385 / 03$ is a new vehicle just $2-3$ months in operation at the time of the evaluation so considered still to be in a "breaking in" phase.

Table 3: A summary table from phase 3 of the fuel enhancement evaluation at Letšeng Diamond Mine, showing the fuel economy figures in litres per hour of the caterpillar fixed-head dump trucks before and after initiation of Product $\mathrm{C}$.

\begin{tabular}{|c|c|c|c|c|c|c|c|c|}
\hline & Baseline & \multicolumn{3}{|c|}{ First 10 Days } & \multicolumn{3}{c|}{ Last 10 Days } & $\%$ Change \\
to Baseline
\end{tabular}


Note 1: Since the baseline fuel consumption information of the electronic system and the manual recording system did not have a large variance, the results of the final stage of phase 3 was compared to the baseline, as this recording of consumption data was reliable and valid.

Table 4: A summary table from phase 3 of the fuel enhancement evaluation at Letšeng Diamond Mine, showing the improvement in smoke figures in percentage of the caterpillar fixed-head dump trucks before and after initiation of Product $\mathrm{C}$.

\begin{tabular}{|c|c|c|c|}
\hline EQUIPMENT & BASELINE & FINAL & $\%$ Change \\
\hline $773-R 01$ & 19.8 & 12.7 & $\mathbf{- 3 5 . 8 0 \%}$ \\
\hline 773-R02 & 23.9 & 16.9 & $\mathbf{- 2 9 . 4 0 \%}$ \\
\hline $\begin{array}{c}\text { FLEET } \\
\text { AVERAGE }\end{array}$ & $\mathbf{2 1 . 9}$ & $\mathbf{1 4 . 8}$ & $\mathbf{- 3 2 . 3 0 \%}$ \\
\hline
\end{tabular}

Upon agreement of success of fuel consumption and emissions reductions, a phase 2 optical survey was permitted. The results of this can be seen most significantly in figure 1 and figure 2 . The elimination of exhaust black smoke and soot clearly demonstrates that the use of product $\mathrm{C}$ created a cleaner, more efficient fuel combustion.

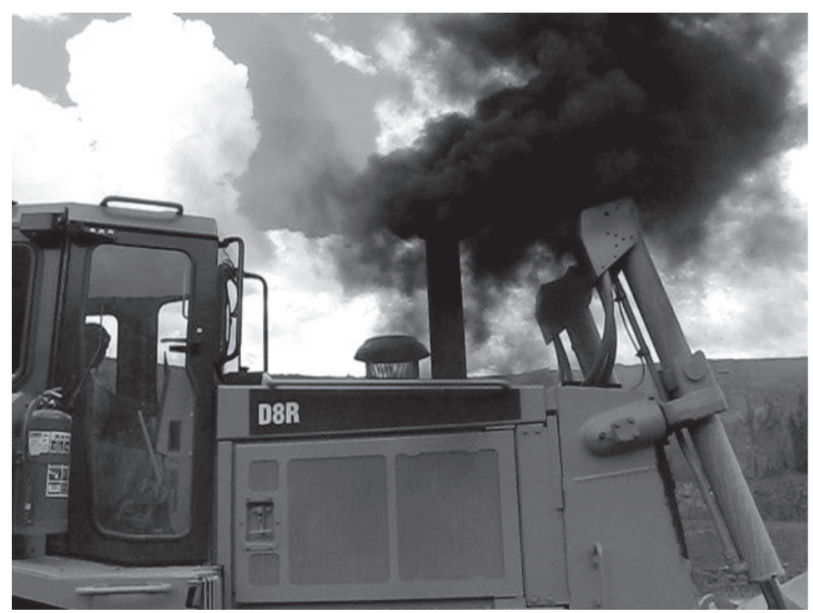

Figure 1: Optical presentation of Phase 2 of the fuel enhancement evaluation at Letšeng Diamond Mine before addition of product $\mathrm{C}$.

Phase 3 was performed as a final proof of encouraging results. During this phase, consumption, visual and emission reductions were monitored on the fixed-head dump trucks. Tables 3, 4 and 5 give the results of phase 3 . When working with diesel the interest lies mainly with NOx as well as PM. Table 5 reflects the results specifically of NOx and opacity, which is an indicator of PM. Product $\mathrm{C}$ should have reduced the other harmful components as well as reducing NOx and opacity. The results shown in table 5 indicate an improved combustion process. An improved combustion process should show a reduction in $\mathrm{CO}, \mathrm{HC}$ and a minor increase in $\mathrm{CO}_{2}$, resulting in less overall harmful emissions, improved air quality and fuel economy performance. A marked improvement in the performance and ease of operability of the engines due to cleaner burning fuel was also noticed.

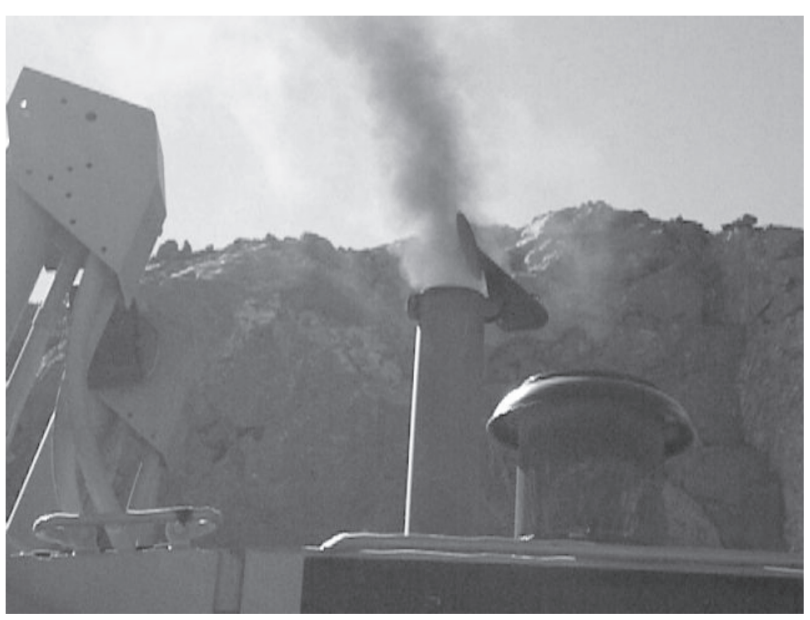

Figure 2: Optical presentation of the reduction in smoke 21 days after addition of product $C$ during the fuel enhancement project at Letšeng Diamond Mine.

However when referring to table 5, there was an increase in $\mathrm{HC}$ and $\mathrm{CO}$ with a reduction in $\mathrm{CO}_{2}$. This is rather puzzling as these results do not correspond with the improved consumption and reduced NOx and opacity, according to SAE J1667 methodology. This aspect of inconsistency is still being researched. Essentially the reduction in all the harmful diesel exhaust emissions was not realised. Table 5 on next page. 
Table 5: Summary table from phase 3 of the fuel enhancement evaluation at Letšeng

Diamond Mine, showing the emission improvement on the caterpillar fixed-head dump trucks before and after the initiation of Product $\mathrm{C}$.

\begin{tabular}{|c|c|c|c|c|c|c|c|c|c|c|c|c|c|c|c|c|}
\hline \multicolumn{17}{|c|}{ Baseline Emissions - March 4th, 2010} \\
\hline \multirow{2}{*}{$\begin{array}{c}\text { EQUIP } \\
\text { NUMBER }\end{array}$} & \multicolumn{5}{|c|}{ Emissions-Elevated RPM } & \multicolumn{5}{|c|}{ Emissions -Idle } & \multicolumn{5}{|c|}{ Average Emissions } & \multirow{2}{*}{$\begin{array}{c}\text { Opacity } \\
(\%)\end{array}$} \\
\hline & $\mathrm{HC}$ & $\mathrm{co}$ & $\mathrm{CO} 2$ & 02 & Nox & $\mathrm{HC}$ & $\mathrm{co}$ & $\mathrm{CO} 2$ & 02 & Nox & $\mathrm{HC}$ & $\mathrm{CO}$ & $\mathrm{CO} 2$ & 02 & Nox & \\
\hline 773-R01 & 4 & 0.031 & 5.28 & 13.67 & 425 & 4 & 0.018 & 2.82 & 17.08 & 386 & 4 & 0.025 & 4.05 & 15.38 & 406 & 19.8 \\
\hline 773-R02 & 2 & 0.038 & 5.38 & 13.46 & 436 & 3 & 0.019 & 2.67 & 17.15 & 375 & 2 & 0.029 & 4.03 & 15.30 & 406 & 23.9 \\
\hline 773-R03 & 5 & 0.031 & 5.51 & 13.31 & 455 & 6 & 0.016 & 2.73 & 17.14 & 365 & 5 & 0.024 & 4.12 & 15.22 & 410 & $\mathrm{n} / \mathrm{a}$ \\
\hline FLEET AVERAGE & 3 & 0.033 & 5.39 & 13.48 & 439 & 4 & 0.018 & 2.74 & 17.12 & 376 & 4 & 0.026 & 4.07 & 15.30 & 407 & 21.9 \\
\hline
\end{tabular}

\begin{tabular}{|c|c|c|c|c|c|c|c|c|c|c|c|c|c|c|c|c|}
\hline \multicolumn{17}{|c|}{$\%$ Change in Emissions } \\
\hline \multirow{2}{*}{$\begin{array}{c}\text { EQUIP } \\
\text { NUMBER }\end{array}$} & \multicolumn{5}{|c|}{ Emissions-Elevated RPM } & \multicolumn{5}{|c|}{ Emissions - Idle } & \multicolumn{5}{|c|}{ Average Emissions } & \multirow{2}{*}{$\begin{array}{c}\text { Opacity } \\
\text { (\%) }\end{array}$} \\
\hline & $\mathrm{HC}$ & $\mathrm{co}$ & $\mathrm{CO} 2$ & 02 & Nox & $\mathrm{HC}$ & $\mathrm{co}$ & $\mathrm{CO} 2$ & 02 & Nox & $\mathrm{HC}$ & $\mathrm{co}$ & $\mathrm{CO} 2$ & 02 & Nox & \\
\hline 773-R01 & $27 \%$ & $11 \%$ & $-2 \%$ & $1 \%$ & $-4 \%$ & $31 \%$ & $13 \%$ & $-4 \%$ & $1 \%$ & $-5 \%$ & $29 \%$ & $12 \%$ & $-3 \%$ & $1 \%$ & $-5 \%$ & $-36 \%$ \\
\hline 773-R02 & $200 \%$ & $-4 \%$ & $-9 \%$ & $5 \%$ & $-16 \%$ & $100 \%$ & $-4 \%$ & $1 \%$ & $0 \%$ & $-3 \%$ & $136 \%$ & $-4 \%$ & $-6 \%$ & $2 \%$ & $-10 \%$ & $-29 \%$ \\
\hline 773-R03 & $n / a$ & $n / a$ & n/a & $\mathrm{n} / \mathrm{a}$ & $n / a$ & $\mathrm{n} / \mathrm{a}$ & $\mathrm{n} / \mathrm{a}$ & $\mathrm{n} / \mathrm{a}$ & $\mathrm{n} / \mathrm{a}$ & $n / a$ & $n / a$ & $n / a$ & $\mathrm{n} / \mathrm{a}$ & $n / a$ & $n / a$ & n/a \\
\hline LEET AVEF & $45 \%$ & $7 \%$ & $-7 \%$ & $4 \%$ & $-12 \%$ & $31 \%$ & $9 \%$ & $-1 \%$ & $0 \%$ & $-3 \%$ & $37 \%$ & $7 \%$ & $-5 \%$ & $2 \%$ & $-8 \%$ & $-32 \%$ \\
\hline
\end{tabular}

\section{Discussion}

The aim of the study was to compare the effects of fuel additives on harmful exhaust emissions and fuel consumption. The obtained results do indicate that not all fuel additives are worth considering because some have severe immediate effects on diesel powered engines, for example, product $B$, and possible long-term effects on upper cylinder engine parts e.g. product A (discussed below).

Product A was a locally blended alcohol based product with claims of reducing consumption and harmful emissions. It was stated as a combustion enhancer. Product B was also a locally blended paraffin based product with claims of being biodegradable and environmentally friendly. Neither of the products A or B had any proof of testing or certification of any laboratory or field tests performed. Neither of the suppliers of product A or B could supply Material Safety Data sheets. Product $C$ was a USA blended marine seal oil based product, with EPA certification and an updated relevant Material Safety Data Sheet (MSDS). It was said by the manufacturer's to be a biodegradable, environmentally friendly fuel enhancing product.

Table 1 details the consumption results of product $A$. The measurements have been performed over a period of a week. The consumption improvement was $2-3 \%$ over the range of the Caterpillar articulated dump trucks. This marginal improvement cannot be ignored; however it does not seem realistic when return on investment is a goal. A break-even point of fuel saved and cost of the product needs to be attained in order for the product to pay for itself. Although marginal savings were realised the use of the product was discontinued based on research of the effects of alcohol on diesel engines. It was mentioned in various reports and studies that alcohol reduces the lifespan of vehicles. Discontinuation of product $A$ was a result of risk management. The product being alcohol based does not supply a good enough reason to implement the product as concerns of long-term use of a product such as this has a high possibility of reduction in lifespan of the vehicles as alcohol is not complimentary to or compatible with upper cylinder engine parts. Environmental savings are not displayed as there were marginal savings realised during use of this product.

It is however very exciting to see that the study showed that product $\mathrm{C}$ showed improved efficiency in terms of consumption and harmful emissions from diesel exhausts, which are a reduction in NOx and opacity. The discrepancy in $\mathrm{CO}, \mathrm{Co}_{2}$ and $\mathrm{HC}$ should be further investigated. However efficiency, NOx and opacity seem to correlate with the claims of the manufacturer of product $\mathrm{C}$ whereas $\mathrm{CO}, \mathrm{CO}_{2}$ and $\mathrm{HC}$ need futher investigation.

It is suspected that the extreme weather conditions have had a great effect on these results. These conditions are those of high altitude, extreme cold and wind and changing barometric pressure. In the uture there will be mines and other industries interested in running tests similar to this study 
performed so it will be interesting to see the results of the associated research.

\section{Conclusion}

Positive results of just one of the fuel additives tested indicate that further studies should be performed on fuel additives. This is very positive and encouraging. The use of fuel additives cannot be ignored when dealing with diesel pollution means to reduce harmful emissions. Further research should be dedicated to and performed on certain fuel additives as they do have a significant impact on harmful emissions.

\section{Acknowledgments}

This work was financially supported by Letšeng Diamond Mine, Lesotho.

\section{References}

Sarvi A, C-J Fogelholm, R Zevenhoven (2008): Emissions from large-scale medium speed diesel engines: 1. Influence of engine operation mode and turbocharger. Finland: Fuel Processing Technology; $510-519$.

Sarvi A, R Zevenhoven (2009): Large-scale diesel engine emission control parameters. Energy: $\mathrm{XXX} ; 1$ -7 .

Rakopoulos C D, D C Rakopoulos, E G Giakoumis, D C Kyritsis (2004): Validation and sensitivity analysis of a two zone diesel engine model for combustion and emissions prediction. Energy Conversion and Management: 15;1471-1495.

Yanfeng G, L Shenghua, G Hejun, H Tiegang, Z Longbao (2006): A new diesel oxygenate additive and its effects on engine combustion and emissions. Applied Thermal Engineering: 27;202-207.

Armas O, K Yehliu, A L Boehman (2010): Effect of alternative fuels on exhaust emissions during diesel engine operation with matched combustion phasing. Fuel: 89;438-456.

Wong O, R W Morgan, L Kheifets, S R Larson, M D Whorton, (1985): Mortality among members of heavy construction equipment operators with potential exposure to diesel exhaust emissions. British Journal of Industrial Medicine: 42;435-448.

Ouenou-Gamo S, M Ouladsine, A Rachid (1998): Measurement and prediction of diesel engine exhaust emissions. ISA Transactions: 3;135-140. 